\title{
LE STRUCTURALISME FONCTIONNEL PRAGOIS FACE AU STALINISME : BOULEVERSEMENTS, SOUBRESAUTS ET PARADOXES
}

\author{
Ondřej PEŠEK \\ Université de Bohême du Sud, České Budějovice
}

\begin{abstract}
En): In the following paper we focus on the controversial period around the year 1950 in Czechoslovakia which was marked by the advent of the Stalinist regime. Linguistics as well as other sciences and spheres of Czech society had to adapt to the practices of the new totalitarian regime. By means of discourse analysis, we try to show different aspects of the campaign which was led against functional structuralism and, consequently, against the Prague Linguistic Circle in the name of Marxist ideology.
\end{abstract}

Keywords (En): Prague Linguistic Circle; History of linguistics; Functional structuralism

Mots-clés (Fr): Cercle Linguistique de Prague ; histoire de la linguistique , Structuralisme fonctionnel

\section{Introduction}

La fin des années 40 et le début des années 50 en Tchécoslovaquie sont marqués par l'avènement du stalinisme à l'état (presque) pur. Toutes les structures de la société civile se trouvent bouleversées par les pratiques du nouveau régime totalitaire dont l'architecture est orchestrée par Moscou. La linguistique, naturellement, n'en est pas épargnée. Ce bouleversement qui, à cause de son caractère radical et irréductible, ne pouvait pas ne pas être conflictuel, peut s'observer à trois niveaux :

a) au niveau idéologique : la seule opinion tolérée étant la vérité marxisteléniniste, toutes les sciences doivent se conformer à cette nouvelle donne et se voient obligées de redéfinir leurs bases et leurs programmes selon les principes du matérialisme dialectique.

b) au niveau structurel : comme toutes les sciences, la linguistique est portée par les structures universitaires et par les structures savantes du type associatif. Puisque l'emprise du nouveau régime sur la vie de l'État et de ses citoyens est totale, ces structures, elles aussi, lui sont subordonnées et subissent pleinement son pouvoir.

c) au niveau humain: le rôle de l'individu dans le système totalitaire tchécoslovaque est particulier et peut prendre plusieurs formes : collaboration agressive, collaboration conciliante, soumission passive, indifférence, rejet tacite ou résistance ouverte. Aussi paradoxal que cela puisse paraître, le rôle que joue l'individu dans un système totalitaire qui vise la désindividualisation, peut être déterminant pour le déroulement des événements concrets.

De ce point de vue, l'analyse de l'impact qu'avait la réception de l'idéologie et des pratiques stalinistes sur le structuralisme fonctionnel du Cercle linguistique de Prague est exemplaire et en quelque sorte emblématique, car elle implique tous les trois domaines susmentionnés d'une façon édifiante.

Cependant, dresser le bilan de ce conflit représente une tâche épineuse et si l'on se propose pour autant de le faire, on risque de se heurter à de nombreux 
obstacles. Premièrement au niveau de la communauté linguistique et des relations humaines y existant : bien que les événements en questions aient eu lieu il y a plus de soixante ans, leurs conséquences sont toujours sensibles à l'heure actuelle et continuent de façonner, ne serait-ce que très indirectement, les rapports et les choix de la linguistique tchèque contemporaine. Deuxièmement au niveau de l'analyse elle-même: dans les textes datant de l'époque staliniste, l'idéologie est instrumentalisée par les intérêts personnels des protagonistes à tel point que les éléments constitutifs du conflit se trouvent emmêlés dans un discours combatif et déterminé. En interprétant ce discours pour les besoins historiographiques, le risque de fausser la vérité est particulièrement élevé, si tant est que le travail d'historien puisse jamais aboutir à une vérité quelconque.

Étant donné ce caractère problématique du sujet traité, notre objectif doit nécessairement être modeste : loin de vouloir mettre à jour les arrière-plans et les mobiles occultés des joutes idéologiques, nous essaierons avant tout d'analyser et de mettre en perspective les éléments du discours linguistique de l'époque afin de montrer à quel point il était marqué par la situation politique et sociale respective. Néanmoins, nous tâcherons de compléter cette analyse par une évaluation prudente du rôle que jouaient les acteurs principaux des événements relatés. Si notre évaluation peut ressortir de nos interprétations personnelles des indices véhiculés par les textes analysés, nous nous garderons soigneusement de juger les comportements des linguistes engagés.

Notre étude, qui se veut historique, représente une contribution à l'histoire du structuralisme fonctionnel pragois et de son forum institutionnel qu'est le Cercle linguistique de Prague. Cette histoire, comme d'ailleurs toute histoire des idées linguistiques digne de ce nom, ne peut se faire qu'à travers l'analyse des textes qui étaient à la base de la formation socio-discursive en question. Toutefois, pour comprendre les enjeux de ces textes, une présentation du cadre socio-politique externe s'avère nécessaire. La première partie de notre étude sera donc consacrée au cadre social dans lequel évoluait le Cercle linguistique de Prague entre 1945 et 1948. Ce n'est qu'ensuite que nous aborderons la période staliniste et les événements qui y sont associés.

\section{Le Cercle linguistique de Prague et la linguistique tchécoslovaque entre 1945 et 1948}

Pendant la Deuxième Guerre mondiale, le Cercle linguistique de Prague a toujours continué ses activités, quoique d'une manière assez réduite et seulement dans la mesure tolérée par l'occupant nazi. Certes, les membres (et amis) du Cercle se réunissaient régulièrement pour écouter des conférences ${ }^{1}$ et poursuivaient leurs activités de publication ${ }^{2}$, mais ils le faisaient dans une atmosphère de peur et de censure omniprésente. Pour étouffées et opprimées par le régime nazi que fussent les activités scientifiques et citoyennes,

\footnotetext{
${ }^{1}$ Entre mars 1939 et mai 1945, J. VACHEK (1999) compte plus de 60 conférences prononcées dans le cadre du Cercle.

${ }^{2}$ Rappelons les forums publicationnels du Cercle les plus importants : la revue Slovo a slovesnost, qui continue à paraître jusqu'en 1943 et l'ouvrage collectif Čtení o jazyce a poezii paru en 1942. 
le Cercle linguistique de Prague a réussi à survivre aux horreurs de la guerre et croyait retrouver, en 1945, une paix et une liberté durables ${ }^{3}$.

Après la libération, le Cercle désire dynamiser ses activités et notamment renouer avec les échanges internationaux. Il profite de l'ouverture des frontières et invite de nombreux conférenciers étrangers qui, reconnaissant le prestige dont jouissait le Cercle parmi les linguistes, ne refusent jamais d'accepter cette invitation ${ }^{4}$. Entre 1945-48, le Cercle poursuit ses activités éditoriales, organise activement des conférences ${ }^{5}$, participe collectivement aux congrès ${ }^{6}$ et continue de représenter la référence la plus prestigieuse de la science linguistique tchécoslovaque.

Pourtant, malgré cette volonté affichée de renouer avec les meilleures traditions du Cercle d'avant 1939, l'atmosphère sociale n'est plus du tout la même qu'avant la guerre et d'aucuns ne manquent pas de le souligner dans leur correspondance. Citons à titre d'exemple l'extrait de la lettre qu'écrivait Antonín Mágr $^{7}$ à R. Jakobson en novembre 1945 : «Le Cercle n'est plus le vieux Cercle de votre époque et de celle de Mathesius» (cité selon J. TOMAN, 2011 : 269). Cette remarque ne pourrait certes être interprétée que comme un regret personnel concernant le climat des débats au sein du Cercle, car le rôle de Jakobson et Mathesius y était déterminant, mais nous croyons que cette observation faite dès 1945 laisse présager des changements d'une tout autre nature. En effet, tout au long de la période mai 1945 - février 1948, nous enregistrons des signaux inquiétant qui montrent que la liberté regagnée n'était que très relative. Le «cas Jakobson » est particulièrement édifiant à ce sujet. Après la guerre, le Cercle désire le retour de son éminent représentant, qui a réussi entre-temps à se faire une excellente réputation dans le milieu scientifique américain. L'Université Masaryk de Brno, où Jakobson enseignait avant la guerre, a expressément confirmé que son poste de professeur était vacant et qu'il était toujours réservé pour lui. Selon J. VACHEK (1999), la présidence du Cercle a consulté l'ambassade soviétique pour connaître son avis quant au retour de R. Jakobson en Tchécoslovaquie; cet acte est significatif en lui-même puisque Jakobson était, depuis 1937, citoyen tchécoslovaque. En réagissant à cette demande, l'ambassadeur Zorin a recommandé que Jakobson ne revienne pas tout de suite, il a conseillé qu'on «attende». Cet épisode montre clairement que dès

\footnotetext{
${ }^{3}$ Il a certes perdu trois de ses représentants les plus éminents : V. Mathesius (mort le 12 avril 1945), N. S. Troubetzkoy (mort le 25 juin 1938) et R. Jakobson (émigration clandestine en avril 1939), mais leur disparition ne pouvait pas freiner l'enthousiasme et l'optimisme avec lesquels les structuralistes pragois envisageaient leur avenir en mai 1945.

${ }_{4}^{4}$ Ainsi en 1946, le Cercle accueille à Prague S. Urbańczyk et en 1947, A. S. C. Ross, L. Hjelmslev, E. J. Simmons, M. R. Mayenowa et G. Devoto.

${ }^{5}$ Selon les données de J. VACнек (1999), entre mai 1945 et février 1948, 28 conférences ont été prononcées dans le cadre du Cercle.

${ }^{6}$ Nous pensons notamment au Congrès international des linguistes qui se tenait à Paris en juillet 1948. La date du congrès est certes postérieure au 25 février 1948, mais les linguistes pragois préparaient leurs réponses collectives bien avant que les communistes ne s'emparent du pouvoir en Tchécoslovaquie.

${ }^{7}$ Antonín Stanislav Mágr était un journaliste à Prager Presse. Dans ses essais d'avant-guerre, il faisait souvent référence aux travaux de R. Jakobson.
} 
1945, les Soviétiques avaient une forte influence sur la politique intérieure tchécoslovaque. Pour eux, Roman Jakobson, juif d'origine et émigré chez les « impérialistes », était forcément une personna non grata, ce qu'ils n'hésitaient pas à faire savoir aux autorités tchécoslovaques. On sait d'ailleurs qu'après l'entrée de l'armée rouge sur le territoire tchécoslovaque, de nombreux émigrés russes et ukrainiens ont été arrêtés et déportés en URSS par les agents soviétiques $^{8}$. Il était donc évident que la sécurité de Jakobson dans la Tchécoslovaquie libérée ne pouvait pas être pleinement assurée : il a préféré, sagement, rester aux États-Unis.

Ces événements illustrent le fait bien connu des historiens de l'époque moderne : entre 1945 (et plus particulièrement depuis les élections en mai 1946) et février 1948, la liberté et l'indépendance dont jouissait à l'extérieur l'état tchécoslovaque n'était qu'apparente. Les communistes, soutenus par Moscou, exerçaient pleinement leur influence qui se faisait sentir dans toutes les sphères de la vie publique.

Étant donné ce climat social, les linguistes tchèques sentent que désormais, bien plus qu'avant, il est opportun de suivre de près les orientations de la science soviétique et de se positionner par rapport à elle. C'est dans ce contexte politique que s'effectue la réception de l'article de N. S. TCHEMODANOV, publié en 1947 dans les Izvestiya Akademii Nauk SSSR. Il s'agit d'une critique idéologique du structuralisme qui est présenté par Tchemodanov comme un courant idéaliste et bourgeois. N. S. Tchemodanov s'en prend surtout aux bases saussuriennes du structuralisme qu'il évalue du point de vue de la linguistique marriste ${ }^{9}$. Sa critique vise notamment la conception structuraliste de la langue selon laquelle cette dernière serait une structure immanente sans lien à la pensée, à l'homme et à l'évolution de la société. Le structuralisme réduit ainsi la linguistique à une science purement formelle, agnostique, qui exclut de son champ d'études la réalité extralinguistique. Poussant à l'extrême la conception abstraite et logique du système, le structuralisme reste incapable d'expliquer les tendances évolutives de la langue. Contre ce structuralisme anti-social et anti-historique, Tchemodanov avance les exploits de la linguistique marriste qui, elle, est apte à donner à la linguistique une nouvelle orientation exempte des failles critiquées. Les linguistes tchèques, conscients déjà de l'influence décisive de la force soviétique, se sentent obligés de réagir. Rappelons qu'en 1947, le structuralisme fonctionnel pragois continuait de représenter la doctrine la plus influente dans le milieu scientifique tchécoslovaque : il était donc clair que si le texte de Tchemodanov restait sans réponse, les positions du structuralisme en Tchécoslovaquie pouvaient être sérieusement menacées. C'est donc dans ce contexte que V. SKALIČKA écrit son fameux article ${ }^{10}$ Le structuralisme de Copenhague et l'École de Prague dans

\footnotetext{
${ }^{8}$ Rappelons à titre d'exemple le cas de Piotr Savitski, membre du Cercle de Prague, qui a été déporté en URSS en 1945. Il n'est rentré à Prague qu'en 1956.

${ }^{9}$ Rappelons qu'à cette époque, le marrisme était la doctrine officielle en URSS, selon ses partisans la seule doctrine véritablement marxiste.

10 Le texte, qui a été publié en 1948 dans Slovo a slovesnost, est la version écrite de la conférence que Skalička a prononcée au Cercle linguistique de Prague le 20 octobre 1947.
} 
lequel il essaie de montrer que les reproches formulés par Tchemodanov ne concernent que le structuralisme danois et que par conséquent, le structuralisme pragois échappe aux critiques du linguiste soviétique. Ainsi, Skalička ne contredit pas le fond des critiques, mais essaie de montrer que le structuralisme fonctionnel pragois diffère du programme hjelmslevien et doit donc être traité à part. Il souligne avant tout que dans la conception pragoise, la langue n'est pas purement une structure immanente, puisqu'elle est déterminée par ses fonctions. Celles-ci sont à comprendre comme des tâches que remplit la langue dans la communication: elles assurent ainsi son ancrage dans la société. De même, l'adage saussurien la langue est une forme et non une substance, qui ouvre la voie aux accusations du formalisme, n'a pas de pertinence pour la linguistique pragoise : pour les Pragois, les signes sont toujours considérés dans leur rapport à la réalité, ce qui permet à $\mathrm{V}$. Skalička de se démarquer contre la sémiotique formaliste hjelmslévienne. Pour échapper aux accusations d'anti-socialisme, Skalička souligne que les membres du foyer pragois ont toujours étudié les fonctions que la langue joue dans d'autres institutions sociales: dans la littérature, dans la culture ou dans l'art. Pour le prouver, il cite les travaux de Jan Mukařovský et de son école. Tout cela donc pour montrer que le structuralisme pragois ne pèche pas par l'idéalisme et qu'il devrait donc être à l'abri des attaques marxistes. Cette tentative de sauver le structuralisme fonctionnel a été vaine. La défense de Skalička (prononcée en 1947 et publiée en 1948) est restée sans effet: ses arguments n'ont pas réussi à faire changer la tendance en URSS : on continuait à ne pas distinguer les différents structuralismes qui restaient tous condamnés comme idéalistes et donc incompatibles avec l'idéologie marxiste. Remarquons un fait important: V. Skalička ne met pas en doute les critiques de Tchemodanov, il essaie de les nuancer pour montrer qu'elles ne s'appliquent pas au structuralisme fonctionnel pragois, qu'il ose encore défendre et soutenir publiquement. Pourtant, au lieu de se demander pourquoi une position idéaliste devrait être critiquée en principe, il s'efforce de montrer que le structuralisme pragois n'est pas idéaliste et pourquoi il ne l'est pas. C'est peut être aussi cette argumentation de V. Skalička qui élargi le fossé séparant la sémiotique pragoise d'après-guerre et le saussurianisme authentique.

\section{L'époque staliniste : 1948-1952}

$\mathrm{Si}$, jusqu'en 1948, le pouvoir soviétique en Tchécoslovaquie agissait d'une manière plutôt cachée et tentaculaire, après le putsch communiste du 25 février, ce pouvoir staliniste est officiellement invité à façonner la vie de son nouveau satellite et en devient, naturellement, d'autant plus agressif. Désormais, tout dans l'état tchécoslovaque doit se conformer au modèle soviétique ${ }^{11}$ sous peine d'exclusion, ostracisme, d'emprisonnement ou de mort.

\footnotetext{
${ }^{11}$ Ce qu'exprimait le slogan omniprésent «L'Union soviétique - notre modèle ». Ce slogan est devenu le sésame de la politique intérieure de la Tchécoslovaquie communiste.
} 


\subsection{Le « flirt marriste »}

Les linguistes, eux aussi, se doivent de suivre le modèle du grand frère qui représente, pour le pouvoir communiste tchécoslovaque, la référence suprême. À tel point qu'ils n'hésitent parfois pas à l'instrumentaliser pour faire passer les politiques que l'URSS staliniste elle-même ne pratiquait guère. Comme nous l'avons $\mathrm{vu}$, le structuralisme (y compris le structuralisme fonctionnel pragois), courant bourgeois et idéaliste, était exclu de la science soviétique, suite aux critiques que lui adressait $\mathrm{N}$. S. Tchemodanov dans son article. La réponse de Skalička s'était montrée insuffisante. Dans ce contexte, il n'était point opportun d'afficher publiquement et fermement sa conviction structuraliste. Aussi les linguistes tchèques, dans leur quête de l'ancrage idéologiquement conforme de leur science, se tournent-ils vers les théories de N. J. Marr qui, elles, se proclamaient marxistes et qui jouissaient, en 1948, du statut de la doctrine linguistique officielle en URSS. Ainsi commence la courte période (1948-1950) pendant laquelle les linguistes pragois ${ }^{12}$ cherchent à trouver des passerelles entre le structuralisme fonctionnel et la «nouvelle doctrine de la langue » de Marr. Nous constatons alors, au sein de la linguistique tchèque, un effort de publication d'inspiration marriste sans précédent. C'est ainsi que Bohuslav HAVRÁNEK, dans son étude de 1948 cherche à concilier les deux courants en essayant d'identifier des points communs et de transposer la terminologie structuraliste à celle du marrisme $^{13}$. Entre 1949 et 1950 nous comptons 4 conférences marristes prononcées au sein du Cercle linguistique de Prague $:^{14}$

- le 7 mars 1949 : C. Bosák - La stadialité dans la langue (Stadiálnost v jazyce);

- le 14 novembre 1949: I. I. Mechtchaninov ${ }^{15}$ - Le développement de la linguistique soviétique (Razvitije sovestago jazykoznanija);

- le 17 avril 1950: A. V. Issatchenko - Les caractéristiques de classe des langues standards (Tř́ídní charakter spisovných jazyků);

- le 24 avril 1950 : V. Skalička - N. J. Marr.

Le forum de publication du Cercle, la revue Slovo a slovesnost, publie deux articles de I. I. MECHTCHANINOV (1949, 1950); en novembre 1949, parait un recueil d'articles marristes, présenté comme cadeau offert au $\mathrm{IX}^{\mathrm{e}}$ Congrès du Parti communiste tchécoslovaque et dont l'édition a été dirigée (entre autres) par les représentants éminents du CLP ${ }^{16}$. Rappelons aussi les travaux d'A. V. ISSATCHENKO (1949), de B. HAVRÁNEK (1950a), et de F. TRÁVNíČEK (1950a) qui

\footnotetext{
${ }^{12} \mathrm{Ou}$ plus exactement certains linguistes pragois, en l'occurrence les plus influents d'entre eux.

${ }^{13}$ Tâche objectivement assez hasardeuse. Pour Havránek, l'un des points communs est représenté par la reconnaissance du principe de convergence dans le développement des langues.

${ }^{14}$ Ces conférences marristes comptent parmi les 16 conférences prononcées au Cercle entre 19481950 dont l'existence a été récemment mise en évidence par ČERMÁK, P. - POETA, C. - ČERMÁK, J. (2012).

${ }^{15}$ I. I. Mechtchaninov passait pour la plus grande autorité marriste de l'URSS. Son invitation au Cercle était donc un événement de prime importance puisqu'il s'agissait d'une sommité de la linguistique soviétique.

16 Cf. la bilbliographie, Bosák, C; Hausenblas, K. ; Havránek, B. ; Horálek, K. ; SEDLÁČEK, J. ; SKALIČKA, V. (1949)
} 
sont tous guidés par la volonté de faire passer la doctrine marriste dans le milieu tchécoslovaque.

En considérant cet effort de réception du marrisme en Tchécoslovaquie, nous pouvons constater qu'elle prenait en principe deux formes: 1) un effort de conciliation du marrisme avec certaines positions structuralistes (stratégie typique pour B. Havránek par exemple) ou 2) présentation du marrisme comme une doctrine progressiste, mais sans condamner explicitement le structuralisme (position pratiquée par exemple dans les contributions de C. Bosák). Pour résumer, nous dirions que jusqu'en 1951, l'attitude que montraient publiquement les linguistes tchèques par rapport au structuralisme pragois était prudente : il n'y avait pas de confession ouverte de la foi structuraliste, mais il n'y avait pas non plus de condamnations directes et irréductibles. Dans leurs discours, les linguistes proclamaient leur volonté de suivre la voie marxiste, définie et tracée par la référence soviétique, mais ils n'ont pas agi ouvertement, ni agressivement contre le structuralisme linguistique.

\subsection{L'intervention de J. V. Staline et ses conséquences pour la linguistique tchécoslovaque}

Cependant, tout change à partir de la deuxième moitié de l'année 1950. Le tournant est marqué par la publication dans la Pravda de l'article de J. V. STALINE en juin 1950. Le generalissimus entre ainsi dans le débat mené par les éminents représentants de la linguistique soviétique sur les pages du quotidien Pravda tout au long de l'année 1950. Ce débat opposait les partisans du marrisme à leurs critiques et prenait l'allure d'une affaire d'État dont l'ampleur dépassait le cadre modeste de la science linguistique (cf. à ce propos J.-C. DUPAS, 1977). Staline se range décisivement du côté des opposants et condamne, une fois pour toutes, les thèses marristes ${ }^{17}$. Cette intervention historique de Staline était bel et bien motivée politiquement: Staline a profité du débat pour faire passer dans l'opinion publique des éléments de ses visions politiques ${ }^{18}$. L'incompatibilité du marrisme avec la politique intérieure stalinienne se situe notamment à deux niveaux :

1) la doctrine évolutionniste de Marr n'opérait pas avec la notion de «nation ». Ceci était contraire à la position de Staline, qui désirait souligner le rôle directeur de la nation (et de la langue) russe au sein de l'URSS.

2) la linguistique mondiale a démontré à plusieurs reprises les failles scientifiques du marrisme ${ }^{19}$. Staline se rendait compte que de continuer à soutenir le marrisme comme doctrine officielle de son empire aurait des conséquences désastreuses sur la réputation de la science soviétique.

\footnotetext{
${ }^{17}$ Les linguistes tchèques, fidèles au slogan «L'Union soviétique - notre modèle », ont suivi de très près ce débat: $\mathrm{P}$. Sgall en réfère au Cercle linguistique de Prague lors d'une conférence prononcés dès le 12 juin 1950.

${ }^{18} \mathrm{Cf}$. à ce sujet J. TOMAN $(2011: 273$, note 15$)$.

${ }^{19}$ Les aspects critiqués concernaient notamment la théorie de l'évolution stadiale, l'essai de tirer l'origine des langues à partir des 4 éléments et la position phare du marrisme postulant que la langue est un phénomène de classe.
} 
Traitant le marrisme de «vulgarisation marxiste », Staline définit les grands contours d'une nouvelle linguistique moderne, véritablement marxiste. Malgré cette ambition, le programme stalinien reste assez traditionnel, ne reflétant pas ou presque pas les innovations épistémologiques de la première moitié du $\mathrm{XX}^{\mathrm{e}}$ siècle.

La linguistique tchèque ne manque pas de suivre le pas de la linguistique soviétique. Suite à l'intervention de Staline, la vague marriste qui a atteint les linguistes tchèques cherchant à tout prix leur modèle dans l'URSS prend fin. L'exposé de Staline est tout de suite traduit et publié dans le Slovo a slovesnost (num. 2 de l'année 1950) ${ }^{20}$. Les linguistes tchèques ne tardent pas à saluer cette intervention de Staline et s'agitent pour montrer leur adhésion à ses idées : en 1950 V. SKALIČKA (1950), B. HAVRÁNEK (1950b) ou F. TRÁVNíČEK (1950b) dans la Tvorba saluent avec enthousiasme la génialité du camarade Staline et condamnent résolument le marrisme. À la faculté de Lettres de Prague, de jeunes radicaux communistes organisent des discussions stalinistes sur l'apport des idées de Staline à la linguistique contemporaine. Ces discussions, qui se déroulaient dans l'ambiance des épurations idéologiques des universités sévissant à cette époque dans le milieu académique tchèque, ne manquaient pas d'agressivité et d'attaques personnelles. Elles laissaient présager la campagne anti-structuraliste qui a été organisée sur les pages de l'hebdomadaire Tvorba en 1951 et qui avait pour but de liquider tout ce qui avait trait à la tradition structuralefonctionnelle de l'avant-guerre.

La situation était encore une fois paradoxale. Les thèses de Staline étaient antimarristes, il n'y avait rien d'explicitement anti-structuraliste. Mais le régime tchécoslovaque voulait en finir avec la tradition scientifique de l'avant-guerre pour se conformer complètement à la science staliniste contemporaine ; tous les moyens étaient bons pour atteindre cet objectif. Ainsi a-t-on réglé deux choses d'un seul coup : profitant du flirt avec le marrisme, l'argumentation des acteurs de cette campagne anti-structuraliste se basait sur les arguments contre le marrisme utilisés par Staline, assortis des critiques de Tchemodanov (1947) contre le structuralisme, dans le but de démolir la tradition du structuralisme fonctionnel de la Première république tchécoslovaque. Nous nous proposons maintenant d'analyser de plus près le discours de ce débat, en montrant les points communs et les divergences dans l'approche et dans l'argumentation des différents auteurs. Précisons que l'hebdomadaire Tvorba était la principale tribune idéologique du régime communiste, qui se distinguait par un discours combatif, radical et qui pratiquait une attitude irréductiblement hostile à tout ce qui ne relevait pas de l'idéologie officielle. En 1951, nous comptons 8 articles qui font partie de cette fameuse campagne anti-structuraliste (nous les citons dans l'ordre chronologique de leur parution dans Tvorba) :

20 L'année 1950 de la revue témoigne bien des soubresauts idéologiques caractéristiques de l'époque : le numéro 1 de 1950 contient un article marriste de I. I. MEChTCHANiNov et un rapport détaillé sur sa visite en Tchécoslovaquie, alors que le numéro 2 publie la traduction des thèses de J. V. Staline. Le numéro 3-4 de cette ancienne tribune structuraliste apporte un résumé et un commentaire détaillés des thèses staliniennes, rédigés par J. MORAVEC. 
- SGaLl, P. (1951) : «Stalinovy práce o jazykovědě a pražský linguistický strukturalismus. » [Les travaux linguistiques de Staline et le structuralisme linguistique pragois]

- TRÁVNÍČEK, F. (1951a) : «Strukturalismus - nepřítel naší jazykovědy.» [Le structuralisme - l'ennemi de notre linguistique]

- MUKAŘOVSKÝ, J. (1951) : «Ke kritice strukturalismu v naší literární vědě. » [À propos de la critique du structuralisme dans notre science littéraire]

- BĚLIČ, J. (1951): «Překonáním strukturalismu k marxistické jazykovědě. » [Vers la linguistique marxiste par le dépassement du structuralisme]

- SkALIČKA, V. (1951b) : «Ke kritice strukturalismu. » [À propos de la critique du structuralisme]

- TRÁVNíčEK, F. (1951c): «Strukturalistická typologie jazyková.» [La typologie linguistique structuraliste]

- BARNET, V. (1951) : «Ke kritice strukturalismu v naší jazykovědě. » [À propos de la critique du structuralisme dans notre linguistique ]

- NovÁK, M. (1951): «Ke kritice strukturalismu v české estetice.» [À propos de la critique du structuralisme dans l'esthétique tchèque]

Sans en faire plus de commentaires, remarquons que le grand absent de cette campagne offensive était B. Havránek.

L'article de P.Sgall ${ }^{21}$ inaugure ce débat et pose les contours de l'argumentation utilisée dans le cadre de la campagne: la vraie linguistique marxiste dont la voie a été montrée par J. V. Staline ne peut pas être bâtie en Tchécoslovaquie tant qu'on n'aura pas éliminé les éléments nocifs et erronés contenus dans la doctrine structuraliste, qui d'ailleurs présente de nombreux points en commun avec le marrisme. Ainsi s'opère une subversion idéologique assez sophistiquée: l'attaque contre le structuralisme est encadrée par l'autorité de Staline et c'est à son nom et au nom de son intervention contre le marrisme dans la linguistique soviétique qu'on condamne le structuralisme dans la linguistique tchèque (dans ses articles, Staline s'oppose uniquement à Marr et ne parle pas du tout du structuralisme). Le poids des attaques est ainsi infiniment supérieur à la situation de 1947, lorsque V. Skalička réagissait aux critiques antistructuralistes de Tchemodanov comme s'il s'agissait d'un débat entre paires. Quoique les articles diffèrent l'un de l'autre quant à leur ton et quant à leur contenu, ils comportent certains éléments communs qui représentent le gros

\footnotetext{
${ }^{21}$ P. Sgall (*1926), à l'instar de M. Kundera, I. Klíma ou P. Kohout, appartenait à la génération de jeunes intellectuels tchèques qui ont vécu la deuxième guerre mondiale comme un grand traumatisme entraîné, entre autres, par les failles de l'impérialisme capitaliste. Pour eux, l'URSS passait pour le grand libérateur du joug nazi et le régime communiste pour un nouveau modèle sociétal à suivre. Élève de V. Skalička, P. Sgall s'est laissé engager dans le débat en écrivant le premier article de la série. Après la chute du culte staliniste, P. Sgall a été le seul à écrire un article (P. SGALL, 1956) dans lequel il revient sur ce qu'il avait dit en 1951, notamment à propos de R. Jakobson. Linguiste éminent, jouissant d'une renommée mondiale, P. Sgall a tout au long de sa carrière défendu et développé l'héritage du Cercle linguistique de Prague, ce qui lui a valu, après 1968, des poursuites de la part du régime communiste.
} 
de l'argumentation anti-structuraliste de la Tvorba. Ces éléments peuvent être résumés et schématisés comme suit :

- Le structuralisme linguistique est une théorie bourgeoise et idéaliste.

- Elle est idéaliste parce qu'elle professe l'immanentisme de la structure de la langue.

- De par cet immanentisme, elle sépare la pensée de la langue (J. V. Staline, dans ses articles, fait le même reproche au marrisme).

- Expliquant l'évolution des langues par les lois internes de la structure immanente, la théorie structuraliste est déconnectée de l'histoire de la société et du travail (ce qui est contraire à la thèse staliniste postulant un lien intime entre la langue et l'histoire du peuple). Le structuralisme est donc a-historique.

- Considérant la valeur des signes uniquement au sein de la structure et sans prendre en compte leur référence, le structuralisme déconnecte la pensée de la langue (péché marriste sévèrement condamné par Staline), et la langue de la réalité.

- La conception de la langue en tant que système des signes ouvre la voie aux recherches qui projettent cette conception aux autres phénomènes de la réalité (l'art, la littérature) : ainsi se trouvent mêlées la langue et la culture, ce qui mène à la thèse marriste la plus erronée affirmant que la langue fait partie de la superstructure.

- La thèse (éminemment pragoise) sur les langues fonctionnelles brise l'unité de la langue nationale en suggérant que la langue serait formée par des «langues» fonctionnelles autonomes (ceci est un parallèle à la condamnation formelle par Staline de la thèse marriste affirmant l'existence des langues de classes au sein de la langue nationale).

- La fonction esthétique de la langue et l'idée de la langue poétique mènent au formalisme (condamné également par Staline comme un vice marriste). Ces thèses idéalistes et réactionnaires facilitent la propagation des courants littéraires bourgeois et décadents (littératures non engagées, sans idées, déconnectées des mouvements sociaux, tel le surréalisme).

Nous voyons bien à quel point cette argumentation anti-structuraliste de linguistes tchèques était calquée sur l'argumentation anti-marriste de Staline. Étant donné le caractère militant de l'hebdomadaire Tvorba, le ton des articles est en général assez combatif, le vocabulaire et les tournures utilisées sont tributaires du discours staliniste de l'époque. Citons-en quelques exemples, les plus symptomatiques :

F. Trávníček à propos du structuralisme: «...nous étions tous atteints par le poison structuraliste... »

V. Skalička à propos du structuralisme: «Le structuralisme est sans doute l'enfant de la première république, de l'époque où la science bourgeoise aboutit à un jeu autotélique et à l'obscurantisme servant la classe dirigeante. »

F. Trávníček à propos de Staline : «... tête d'un état socialiste immense, son grand créateur, vainqueur célèbre $d u$ nazisme et $d u$ fascisme dans 
la Grande Guerre patriotique, patron de la paix mondiale et enseignant génialissime de toute l'humanité. »

$\mathrm{V}$. Barnet à propos $\mathrm{du}$ structuralisme: «Après la publication des articles géniaux du camarade Staline, dans lesquels les linguistes ont obtenu un exposé complexe à propos des questions linguistiques fondamentales, mené du point de vue de la science marxiste, nous voyons aujourd'hui clairement que le structuralisme appartient sans aucun doute au passé. »

P. Sgall à propos de R. Jakobson: "Mais dans le Cercle linguistique de Prague s'est insinué un élément étranger, représenté avant tout par un émigré antisoviétique, cosmopolite et trotskiste caché, véritable malfaiteur de notre linguistique, Roman Jakobson, qui trompait beaucoup de nos linguistes, qui les dirigeait dans une mauvaise voie et qui jouait dans la linguistique le même rôle que jouait Karel Teige dans la science et la critique littéraires. »

En considérant certaines de ces tournures, on voit bienque ces articles ressortissent clairement de la propagande, dont le style et les moyens prenaient le dessus sur le fond scientifique. D'ailleurs, en comparant ce discours avec les autres écrits des mêmes linguistes, d'aucuns pensent que c'était la rédaction de l'hebdomadaire Tvorba qui a adapté les textes afin qu'ils soient mieux conformes à son discours habituel. ${ }^{22}$

Tous ces articles de la Tvorba ont une structure assez semblable et présentent des topoï caractéristiques du discours idéologique de l'époque. Ces lieux communs peuvent être synthétisés comme suit : 1) on commence par la critique du passé idéaliste et bourgeois (les arguments voir ci-dessus), 2) on enchaîne par l'autocritique (sebekritika ${ }^{23}$ ): «j'ai été dans l'erreur, Staline m'a fait voir ces erreurs, dans lesquelles je ne persiste plus» et 3) on termine par la prise de l'engagement pour l'avenir (závazek): «mon travail futur sera fait conformément aux principes marxistes définis par J. V. Staline».

Malgré leurs points communs, les articles se distinguent tout de même l'un de l'autre quant à leur ton et leur combativité. Si V. Skalička est conciliant, disant que «Les travaux de l'époque structuraliste doivent être abordés avec prudence et d'une manière critique. Mais cela ne veut pas dire qu'il faudrait jeter tout ce qui était écrit à l'époque. Il est clair que ces ouvrages ne contiennent pas que des erreurs...» et P. Sgall reconnaît les mérites des anciens structuralistes (B. Mathesius, B. Havránek, F. Trávníček, V. Skalička) en soulignant que tous les mauvais principes du structuralisme sont d'importation étrangère ${ }^{24}$, Trávníček de l'autre côté est très virulent et irréductible (structuralisme - ennemi de notre linguistique, poison structuraliste), n'hésitant même pas à dénoncer, sur les pages

\footnotetext{
${ }^{22}$ Par exemple J. Toman (2011: 277) croit que les propos de P. Sgall sur R. Jakobson ont été dûment «améliorés » par les propagandistes de la Tvorba.

${ }^{23}$ Nous citons également les termes tchèques dont les termes français sont la traduction. Les termes tchèques sont en effet idéologiquement très marqués et cette coloration propagandiste se perd dans la traduction.

${ }^{24}$ Il vise notamment R. Jakobson. Le fait d'imputer les positions anti-marxistes à ceux qui avaient émigré à l'étranger était une stratégie assez répandue à l'époque, puisqu'elle permettait à ceux qui sont restés de se mettre à l'abri des poursuites éventuelles (cf. aussi J. TOMAN, 2011).
} 
de la Tvorba, les travaux de ses collègues : «Le travail de l'auteur ${ }^{25}$ témoigne du fait que sa typologie ne s'est pas débarrassée des erreurs structuralistes, qu'elle est noyée dans l'idéalisme autant que le structuralisme. » Remarquonsle encore une fois. Il ne s'agit pas ici tant du fait de critiquer le travail de son collègue, chose normale et courante dans la pratique scientifique, il s'agit de le faire précisément sur les pages de la Tvorba et avec le vocabulaire de la propagande communiste. Se voir accusé d'idéalisme dans la Tchécoslovaquie de 1951 pourrait avoir des conséquences particulièrement néfastes pour l'accusé, allant de la perte de son poste jusqu'à l'emprisonnement et les travaux forcés.

Nous avons vu qu'idéologiquement la linguistique tchèque s'était alignée ouvertement sur la voie staliniste. Le structuralisme fonctionnel, même s'il n'était pas directement visé par Staline, s'est vu condamné comme bourgeois et idéaliste. Dans ce sens, la situation tchécoslovaque doit encore une fois être considérée comme paradoxale. Bien que la doctrine linguistique soviétique officielle du début des années 50 n'adhérât pas au structuralisme, ce courant, à part les critiques relativement modérées à la Tchemodanov, n'est jamais devenu la cible d'une campagne haineuse, comme c'était le cas en Tchécoslovaquie. Nous avançons de cela deux explications : 1) Le structuralisme fonctionnel pragois et, à plus forte raison, son vehiculum institutionnel le Cercle linguistique de Prague, était fortement identifié avec les valeurs masarykiennes de la Première république tchécoslovaque. Il arrangeait bien le pouvoir communiste de voir ce courant condamné, car toute réminiscence du passé «bourgeois » représentait une menace potentielle pour la stabilité du régime. 2) Nous sommes de plus en plus persuadé que les événements des années 1950-1951 étaient une résultante d'un jeu de relations personnelles entre les linguistes les plus influents de l'époque qui, ayant instrumentalisé le débat idéologique, tentaient de régler leurs comptes dans leur lutte d'influence. Même si nous pouvons nous faire une idée sur le rôle que les différents acteurs ont joué dans ce drame, nous nous gardons de les formuler ici, car il ne s'agit, au bout du compte, que de conjectures. De plus, les mobiles des différents linguistes qui ont participé à la poursuite idéologique du structuralisme sont extrêmement complexes et ne se laissent pas résumer en quelques phrases condamnatoires.

Quant au Cercle linguistique de Prague, plateforme presque exclusive du structuralisme fonctionnel, il est resté jusqu'en 1950 un forum linguistique de première importance du pays. C'est ainsi qu'il a servi de porte pour l'introduction du marrisme (lors du flirt 1948-1950) et les membres éminents du Cercle ont été les principaux acteurs des événements de 1950-1951. ${ }^{26}$ Mais la loi $68 / 1951$, relative aux organisations volontaires et aux regroupements, a mis fin

\footnotetext{
${ }^{25}$ I.e. V. Skalička et son Type du tchèque (V. SKALIČKA 1951a).

26 À quelques exceptions admirables près, dont, en particulier, B. Trnka ou J. Vachek. Dans ce contexte, nous ne pouvons qu'admirer l'intégrité morale de Bohumil Trnka lorsque nous lisons l'extrait de la lettre qu'il adressait, le 7 novembre 1951, à Henrik Becker : ...Comme vous le savez, le Cercle a été fondé à son époque pour soutenir le structuralisme fonctionnel. Le bien-fondé de ce courant - que moi-même je considère comme absolument pertinent - est actuellement mis en doute dans la presse quotidienne et ailleurs par certains linguistes tchèques, anciens partisans du marrisme... (cité selon Čermák, P. - Poeta, C. - Čermák, J., 2012 : 422).
} 
à ses activités et à son existence officielle. Sans être pour autant officiellement aboli par un acte juridique, il a disparu de la vie publique au moment où l'idéologie officielle condamnait le structuralisme fonctionnel ${ }^{27}$. Le renouveau structuraliste que l'on date à peu près de 1957 appartient déjà à une autre étape de l'histoire de la linguistique tchécoslovaque.

\section{BIBLIOGRAPHIE}

BARNET, V. (1951), Ke kritice strukturalismu v naší jazykovědě [À propos de la critique du structuralisme dans notre linguistique ], Tvorba 20, p. 11771178.

BĚLIČ, J. (1951), Překonáním strukturalismu k marxistické jazykovědě [Vers la linguistique marxiste par le dépassement du structuralisme], Tvorba 20, p. 987-989.

BosÁK, C. (1949), Sovětský nástup proti lingvistickému idealismu. [L'attaque soviétique contre l'idéalisme linguistique], Slovo a slovesnost, 11, p. 132-135.

BosÁK, C. ; Hausenblas, K. ; HaVRÁNeK, B. ; HorÁleK, K. ; SEDlÁČEK, J. ; SKALIČKA, V. (1949), Sovětská jazykověda: překlady vybraných studiú, [La linguistique soviétique : traductions d'études choisies], Praha, Orbis.

ČERMÁK, P. ; ČERMÁK, J. ; POETA, C. (2012), Pražský lingvistický kroužek $v$ dokumentech [Le Cercle linguistique de Prague dans les documents], Praha, Academia.

DuPAS, J.-C. (1977), Pour et contre Marr : les arguments échangés, Langages, $11 / 46$, p. 38-58.

HAVRÁNEK, B. (1948), Naše pojetí slovanské filologie a její dnešní úkoly. [Notre conception de la philologie slave et ses tâches actuelles], Slávia, 18, p. 264268.

HAVRÁNEK, B.(1950a), Tvůrce nové sovětské jazykovědy [Le créateur de la nouvelle linguistique soviétique], Slovanský přehled, 36, p. 33-34.

HAVRÁNEK, B. (1950b), Stalinova stat' a česká lingvistika. [L'article de Staline et la linguistique tchèque], Tvorba, 19, p. 688-689.

HAVRÁNEK, B. (1952), Dva roky po Stalinově geniálním zásahu do vývoje jazykovědy [Deux ans après l'intervention géniale de Staline dans le développement de la linguistique], Slovo a slovesnost, 13, p. 109-117.

IsSATCHENKO, A. V. (1949), Základy materialistickej jazykovedy [Les bases de la linguistique matérialiste], Slovenská reč 15, p. 65-74.

\footnotetext{
${ }^{27}$ Cf. notamment A. MíšKovÁ (2003) qui décrit les tractations liées aux essais d'incorporer le Cercle dans les structures officielles de l'Académie des Sciences. Vu la campagne antistructuraliste de 1951, il était impossible, pour le Cercle, de satisfaire aux dispositions de l'article 9 de la Loi qui stipulait que des associations fondées avant le $1^{\text {er }}$ octobre 1951 ne peuvent continuer à exister que celles dont les activités sont «en conformités avec les intérêts du peuple travailleur ». La dernière conférence prononcée officiellement au Cercle est celle de V. Skalička du 12 mai 1952 (À propos des dialectes).
} 
MECHTCHANINOV, I. I. (1949), „Nové učení o jazyku“ v SSSR v jeho současné vývojové fázi, [«La nouvelle doctrine de la langue» dans sa phase d'évolution actuelle], Slovo a slovesnost, 11, p. 1-7.

MECHTCHANINOV, I. I. (1950), Úloha N. J. Marra ve vývoji sovětské jazykovědy [Le rôle de N. J. Marr dans le développement de la linguistique soviétique], Slovo a slovesnost, 12, p. 1-9.

MíšKOVÁ, A. (2003), Destruktion und Selbstdestruktion des Prager LinguistikZirkels, Prager Strukturalismus: Methodologische Grundlagen / Prague Structuralism. Methodological Fundamentals, éd. NEKULA, M., Heidelberg, Universitätsverlag Winter, p. 209-224.

MoRAVEC, J. (1950), Sovětská jazykověda a jazykovědná diskuse v „Pravdě“, [La linguistique soviétique et la discussion linguistique dans la «Pravda»], Slovo a slovesnost, 12, p. 113-122.

MUKAŘOVSKÝ, J. (1951), Ke kritice strukturalismu v naší literární vědě [À propos de la critique du structuralisme dans notre science littéraire], Tvorba 20, p. 964-965.

NovÁK, M. (1951), Ke kritice strukturalismu v české estetice [A propos de la critique du structuralisme dans l'esthétique tchèque], Tvorba 20, p. 1252.

NovÁK, P. (1991a), K poválečným osudům české lingvistiky [Ä propos des destins de la linguistique tchèque de l'après-guerre], Slovo a slovesnost 52,p. 183-196.

NovÁK, P. (1991b), Konstanty a proměny Havránkových metodologických postojů (se zvláštním zřetelem $\mathrm{k}$ jeho pojetí marxistické orientace v jazykovědě) [Les constantes et les changements dans les attitudes méthodologiques de Havránek (en tenant compte de sa conception de l'orientation marxiste dans la linguistique)], $K$ poctě akademiku B. Havránkovi. Éd. Marie ČECHOvÁ et al., Praha, Univerzita Karlova, p. 2138.

SGALl, P. (1951), Stalinovy práce o jazykovědě a pražský lingvistický strukturalismus [Les travaux linguistiques de Staline et le structuralisme linguistique pragois], Tvorba 20, p. 674-676.

SGALl, P. (1952), Stalinovy práce o jazykovědě a pražský lingvistický strukturalismus [Les travaux linguistiques de Staline et le structuralisme linguistique pragois], Slovo a slovesnost 13, p. 1-11.

SGALl, P. (1956), Ještě k otázkám strukturalismu [Encore une fois à propos des questions du structuralisme], Slovo a slovesnost 17, p. 181.

SKALIČKA, V. (1948), Kodaňský strukturalismus a pražská škola [Le structuralisme de Copenhague et l'École de Prague], Slovo a slovesnost 10, p. 135-142.

SKALIČKA, V. (1950), Stalinův článek a naše jazykovědná tradice [L'article de Staline et notre tradition linguistique], Tvorba, 19, p. 718.

SKALIČKA, V. (1951a), Typ češtiny [Le type du tchèque], Praha, Slovanské nakladatelství.

SKALIČKA, Vladimír (1951b), Ke kritice strukturalismu [À propos de la critique du structuralisme], Tvorba 20, p. 1011-1012. 
STALINE, J. V. (1950a), O marxismu v jazykovědě [À propos du marxisme dans la linguistique], Slovo a slovesnost 12, p. 57-69.

StAline, J. V. (1950b), K některým otázkám jazykovědy [À propos de quelques questions de la linguistique], Slovo a slovesnost 12, p. 70-74.

TCHEMODANOV, N. S. (1947), Strukturalizm i sovetskoe jazykoznanie [Le structuralisme et la linguistique soviétique], Izvestiya Akademii Nauk SSSR - Otdeleniye literatury i jazyka, 6, p. 115-124.

Toman, J. (2011), Příběh jednoho moderního projektu. Pražský lingvistický kroužek, 1926-1948. [L'histoire d'un projet moderne. Le Cercle linguistique de Prague, 1926-1948], Praha, Karolinum.

TRÁVNíčEK, F (1950a), Akademik Marr a jeho směr v jazykozpytě [L'académicien Marr et son courant dans la linguistique], Naše řě 34, p. 1-6.

TRÁVNíČEK, F. (1950b), Stalinova stat' a česká jazyková kultura [L'article de Staline et la culture linguistique tchèque], Tvorba 19, p. 820.

TRÁVNíČEK, F. (1951a), Strukturalismus - nepř́itel naší jazykovědy [Le structuralisme - l'ennemi de notre linguistique], Tvorba 20, p. 893-894.

TRÁVNÍČEK, F. (1951b), Český jazykozpytný strukturalismus ve světle Stalinova učení o jazyce [Le structuralisme linguistique tchèque à la lumière de la doctrine de Staline sur la langue], Praha, Slovanské nakladatelství.

TRÁVNÍČEK, F. (1951c), „Strukturalistická typologie jazyková” [La typologie linguistique structuraliste], Tvorba 20, p. 1125-1126.

TRÁVNíČEK, F. (1953), Stalinská epocha jazykovědy [L'époque stalinienne de la linguistique], Naše řeč 36, p. 129-139.

TRÁVNíČEK, F. (1954), Ke kritice strukturalismu [À propos de la critique du structuralisme], Slovo a slovesnost 15, p. 143-144.

VACHEK J. (1994), Vzpomínky českého anglisty [Mémoires d'un angliste tchèque]. Jinočany, H \& H.

VACHEK J. (1999), Prolegomena $k$ dějinám Pražské školy jazykovědné [Prolégomènes à l'histoire de l'École linguistique de Prague], Jinočany, $\mathrm{H} \& \mathrm{H}$. 